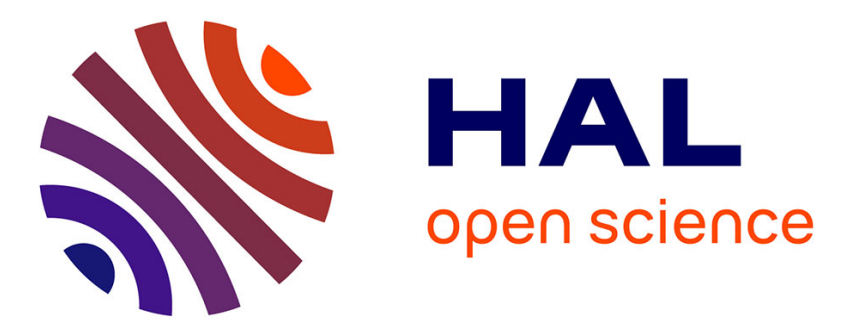

\title{
Analysis of Time and Frequency Domain Pace Algorithms for OFDM with Virtual Subcarriers
}

Christian Rom, Carles Navarro Manchon, Luc Deneire, Troels Sorensen, Preben Mogensen

\section{- To cite this version:}

Christian Rom, Carles Navarro Manchon, Luc Deneire, Troels Sorensen, Preben Mogensen. Analysis of Time and Frequency Domain Pace Algorithms for OFDM with Virtual Subcarriers. IEEE International Symposium on Personal, Indoor and Mobile Radio Communications. PIMRC 2007, Sep 2007, Greece. 5 pp. hal-00290682

\section{HAL Id: hal-00290682 https://hal.science/hal-00290682}

Submitted on 26 Jun 2008

HAL is a multi-disciplinary open access archive for the deposit and dissemination of scientific research documents, whether they are published or not. The documents may come from teaching and research institutions in France or abroad, or from public or private research centers.
L'archive ouverte pluridisciplinaire HAL, est destinée au dépôt et à la diffusion de documents scientifiques de niveau recherche, publiés ou non, émanant des établissements d'enseignement et de recherche français ou étrangers, des laboratoires publics ou privés. 


\section{ANALYSIS OF TIME AND FREQUENCY DOMAIN PACE ALGORITHMS FOR OFDM WITH VIRTUAL SUBCARRIERS}

\author{
Christian Rom, Carles Navarro Manchón, \\ Troels Bundgaard Sørensen and Preben Mogensen \\ Department of Electronic Systems, Aalborg University \\ Niels Jernes Vej 12, Aalborg East, Denmark \\ E-mail: $\{$ chr, cnm, tbs, pm $\} @$ es.aau.dk
}

\author{
Luc Deneire ${ }^{1,2}$ \\ (1) Université de Nice, Sophia Antipolis \\ (2) Centre National de la Recherche Scientifique \\ I3S, UMR 6070, France \\ Email: deneire@i3s.unice.fr
}

\begin{abstract}
This paper studies common linear frequency direction pilotsymbol aided channel estimation algorithms for orthogonal frequency division multiplexing in a UTRA long term evolution context. Three deterministic algorithms are analyzed: the maximum likelihood (ML) approach, the noise reduction algorithm (NRA) and the robust Wiener (RW) filter. A closed form mean squared error is provided for these three algorithms. Analytical and simulation results show that, in the presence of virtual subcarriers, the ML can suffer large performance degradation due to ill-conditioned matrix issues. A solution to this problem is to use the Tikhonov regularization method giving the NRA. The equivalence between the NRA and the RW is proved analytically. A practical implementation of the NRA and RW is proposed based on partial-input partial-output FFT, leading to 6 to 8 times lower complexity than the reference implementation.
\end{abstract}

\section{INTRODUCTION}

Over the last years Orthogonal Frequency Division Multiplexing (OFDM) has been adopted in high data-rates communicathogonality is preserved over the dispersive multipath channel. OFDM is used in upcoming standards such as IEEE 802.16 and UTRA Long Term Evolution (LTE) [1]. Both standards use coherent detection through Pilot-symbol Aided Channel Estimation (PACE) with virtual subcarriers.

Peter Hoeher showed in [2] that the two dimensional interpolation problem of PACE could be solved by using 2 cascaded orthogonal 1-D filters, giving virtually no performance loss compared to the 2-D filter. The latency requirements of standards such as LTE or IEEE 802.16e limit the pilot span available for channel estimation in time direction to a low amount of samples. This increases the importance of the frequency direction interpolation which is the focus of this paper.

Much attention has been given to this topic showing that the performance tradeoff of the algorithms depends on the relationship between the Power Delay Profile (PDP) length and the frequency domain pilot spacing. Deterministic approaches can be separated into time and frequency domain solutions. Deterministic time domain solutions are: the Time Domain Least Squares (TDLS) [3] and [4], The Maximum Likelihood approach (ML) [5], [6] and The Noise Reduction Algorithm (NRA) [7]. Deterministic frequency domain methods are Spline, Gaussian or Lagrange interpolation, and retion systems. By using a Cyclic Prefix (CP) the subcarrier or-

quire higher pilot overhead to achieve an acceptable performance [8]. Bayesian approaches such as the Minimum Mean Squared Error (MMSE) in time domain or frequency domain have been proposed in [2], [3], with complexity reduction by Singular Value Decomposition (SVD) suggested in [9]. However, Bayesian approaches cannot directly be used as they need large channel dependent matrix inversions and require accurate knowledge of channel correlation properties. To reduce their complexity, different solutions have been proposed by, for example assuming a uniform PDP [9], [10].

We propose to study three algorithms: ML, NRA and RW. When introducing virtual subcarriers in the OFDM symbol, unequal MSE distribution at different carriers appears, in particular, MSE increases at band edges [6]. This MSE increase at the band-edges is analyzed, and solutions to alleviate the problem are suggested. The ML approach shows not to be suitable when introducing too many virtual subcarriers, or having channel profiles with large delays. A more convenient approach is to use the NRA by introducing a diagonal matrix in the calculation of the pseudo-inverse of the ML, known as the Tikhonov regularization. The authors prove that the NRA is equivalent to the Robust Wiener (RW) approach when assuming identical channel statistics knowledge at the receiver and a sample spaced PDP. Performance is then evaluated for LTE parameters, and implementation strategies are analyzed focusing on the computational complexity.

The remainder of this paper is structured as follows: the OFDM baseband received signal model is given in section II. An algorithm study is presented in section III, followed by a performance analysis in section IV. The complexity study and implementation strategy are then given in section V. Finally section VI concludes the work.

\section{OFDM BASEBAND RECEIVED SIGNAL}

\section{A. Notations}

The notations used throughout this paper are:

$\begin{array}{ll}\forall & : \text { for all } \\ \in & : \text { membership } \\ (\cdot)^{H} & : \text { hermitian transpose of a matrix or vector } \\ |\cdot| & : \text { absolute value } \\ \operatorname{tr}\{\cdot\} & : \text { trace operator } \\ \mathrm{E}\{\cdot\} & : \text { expectation operator } \\ \mathbf{X}[n, k] & : \text { the } n^{t h} \text { row and } k^{t h} \text { column element of } \\ & \end{array}$


Bold upper-case letters are used for matrices and bold lowercase letters are used for vectors.

\section{B. Received Signal}

In the following an analytical model is derived with the purpose of studying some specific frequency direction channel estimation algorithms. In order to simplify the mathematical expressions of this model, we will assume that the maximum excess delay of the channel is shorter than the $\mathrm{CP}$ and accordingly, assume no Inter-Symbol Interference (ISI) between consecutive OFDM symbols. The channel variations are considered negligible over the duration of 1 OFDM symbol giving Inter-Carrier Interference (ICI) free signal reception. Furthermore, we will consider that the receiver is ideally synchronized with the arrival of the first physical path of the channel. Depending on the initial assumptions different matrix vector models can be derived. Starting with a very general case with all subcarriers used for data transmission, the received signal before channel equalization is:

$$
\mathbf{y}=\mathbf{X F g}+\mathbf{w}=\mathbf{X h}+\mathbf{w}
$$

$\mathrm{X}$ : data symbol diagonal $\left(\mathrm{N}_{f f t} \cdot \mathrm{N}_{f f t}\right)$

F: DFT $\left(\mathrm{N}_{f f t} \cdot \mathrm{N}_{f f t}\right)$

g: Channel Impulse Response (CIR) $\left(\mathrm{N}_{f f t} \cdot 1\right)$

h: Channel Transfer Function (CTF) $\left(\mathrm{N}_{f f t} \cdot 1\right)$

w: Additive White Gaussian Noise (AWGN) $\left(\mathrm{N}_{f f t} \cdot 1\right)$

with $\forall\{n, k\} \in\left[0, \mathbf{N}_{f f t}-1\right]^{2}, \mathbf{F}[n, k]=e^{\frac{-j 2 \pi n k}{\mathbf{N}_{f f t}}}$.

When virtual subcarriers are introduced, data and pilot symbols are only partially using the bandwidth. It is then possible to reorder the rows of $\mathbf{y}$ to have a clear notation that separates virtual subcarriers from the used subcarriers. The perceived CIR is assumed to have a finite length and to be sample spaced. The received vector may be written as:

$$
\mathbf{y}=\left[\begin{array}{cc}
\mathbf{X}_{u} & 0 \\
0 & 0
\end{array}\right]\left[\begin{array}{ll}
\mathbf{F}_{u s} & \mathbf{F}_{u n} \\
\mathbf{F}_{v s} & \mathbf{F}_{v n}
\end{array}\right]\left[\begin{array}{c}
\mathbf{g}_{s} \\
0
\end{array}\right]+\mathbf{w} .
$$

$\mathbf{X}_{u}$ : data and pilot symbol diagonal matrix $\left(\mathrm{N}_{u} \cdot \mathrm{N}_{u}\right)$

$\mathbf{F}_{u s}$ : subDFT of used subcarriers and CIR $\left(\mathrm{N}_{u} \cdot \mathrm{N}_{s}\right)$

$\mathbf{F}_{u n}$ : subDFT of used subcarriers and noise $\left(\mathrm{N}_{u} \cdot\left(\mathrm{N}_{f f t}-\mathrm{N}_{s}\right)\right)$

$\mathbf{F}_{v s}:$ subDFT of virtual subcarriers and CIR $\left(\left(\mathrm{N}_{f f t}-\mathrm{N}_{u}\right) \cdot \mathrm{N}_{s}\right)$

$\mathbf{F}_{v n}$ : subDFT of virtual subcarriers and noise $\left(\left(\mathrm{N}_{f f t}-\mathrm{N}_{u}\right)\right.$.

$\left(\mathrm{N}_{f f t}-\mathrm{N}_{s}\right)$ )

$\mathrm{g}_{s}$ : CIR vector of length of the maximum excess delay $\left(\mathrm{N}_{s} \cdot 1\right)$

$\mathrm{N}_{f f t}$ : FFT size

$\mathrm{N}_{u}$ : number of used subcarriers for data and pilots

$\mathrm{N}_{p}$ : number of pilot subcarries in one OFDM symbol

$\mathrm{N}_{s}$ : number of CIR samples with energy

Only the subset of pilot-carrying subcarriers are available for channel estimation leading to:

$$
\mathbf{y}_{p}=\mathbf{X}_{p} \mathbf{F}_{p s} \mathbf{g}_{s}+\mathbf{w}_{p}=\mathbf{X}_{p} \mathbf{h}_{p}+\mathbf{w}_{p}
$$

where $\mathbf{X}_{p}$ is a diagonal subset matrix of $\mathbf{X}_{u}$ and $\mathbf{F}_{p s}$ is a subset matrix of $\mathbf{F}_{u s}$ where only the elements affecting the pilot subcarriers are considered.

\section{AlgorithM STUDY}

Different classical algorithms are presented in this section. However they are studied in the case of OFDM containing virtual subcarriers which will affect their notation and performance. They are all based on an initial least-squares estimate at the pilot positions. It is noted that the pilots are all transmitted with a M-PSK constellation leading to a constant pilot power. Without loss of generality we assume that this power is set to unity. The initial least-squares estimate at the pilots is given by:

$$
\mathbf{h}_{l s}=\mathbf{X}_{p}^{-1} \mathbf{y}_{p}
$$

\section{A. Maximum Likelihood}

Assuming that the maximum CIR length is known at the receiver, the ML estimate of the channel response is expressed as [5]:

$$
\mathbf{h}_{m l}=\mathbf{F}_{u s} \mathbf{g}_{m l}=\mathbf{F}_{u s}\left(\mathbf{F}_{p s}^{H} \mathbf{F}_{p s}\right)^{-1} \mathbf{F}_{p s}^{H} \mathbf{h}_{l s} .
$$

However, when virtual subcarriers are present, the matrix:

$$
\mathbf{T}=\mathbf{F}_{p s}^{H} \mathbf{F}_{p s}
$$

can become ill-conditioned, leading to high MSE, as it will be shown in Section IV.

\section{B. Noise Reduction Algorithm}

A simple solution to alleviate the ill-conditioning problem is to add a small value $\gamma$ to the diagonal of the matrix $\mathbf{T}$, also known as the Tikhonov regularization method. This is suggested in [7], giving the NRA algorithm:

$$
\mathbf{h}_{n r a}=\mathbf{F}_{u s} \mathbf{g}_{n r a}=\mathbf{F}_{u s}\left(\mathbf{F}_{p s}^{H} \mathbf{F}_{p s}+\gamma \mathbf{I}\right)^{-1} \mathbf{F}_{p s}^{H} \mathbf{h}_{l s} .
$$

Assuming that $\mathbf{F}_{p s}=\mathbf{U} \Delta \mathbf{V}^{H}$ by SVD, with $\lambda_{i}$ being the $i^{t h}$ singular value of $\mathbf{F}_{p s}$, (7) can be rewritten as:

$$
\mathbf{h}_{n r a}=\mathbf{F}_{u s} \mathbf{V} \Delta_{n r a} \mathbf{U}^{H} \mathbf{h}_{l s}
$$

where $\Delta_{n r a}[i, j]=\frac{\lambda_{i}}{\lambda_{i}^{2}+\gamma}$ for $(i=j)$, and $\Delta_{n r a}[i, j]=0$ for $i \neq j$.

\section{Wiener and Robust Wiener}

In his work, Hoeher showed that the optimum linear estimator, in the MSE sense, in PACE OFDM is the Wiener filter, which is given by [2]:

$$
\mathbf{h}_{w}=\mathbf{R}_{\mathbf{h h}_{p}}\left(\mathbf{R}_{\mathbf{h}_{p} \mathbf{h}_{p}}+\sigma_{w}^{2} \mathbf{I}_{N_{p}}\right)^{-1} \mathbf{h}_{l s}
$$

where $\mathbf{R}_{\mathbf{h h}_{p}}$ is the cross-correlation matrix of $\mathbf{h}$ and $\mathbf{h}_{p}$, $\mathbf{R}_{\mathbf{h}_{p} \mathbf{h}_{p}}$ is the autocorrelation matrix of $\mathbf{h}_{p}$ and $\sigma_{w}^{2}$ is the power of the gaussian noise. Generally, the frequency correlation properties are not known at the receiver, and furthermore they can vary over time. For this reason, a robust design based on the assumption of a uniform PDP with sample-spaced equally-powered taps and the same length of the CIR is proposed. The resulting constant correlation matrices can be 
then expressed as:

$$
\mathbf{R}_{\mathbf{h h}}=\mathrm{E}\left\{\mathbf{h h}_{p}^{H}\right\}=\frac{1}{N_{s}} \mathbf{F}_{u s} \mathbf{I}_{N_{s}} \mathbf{F}_{p s}^{H}
$$

and

$$
\mathbf{R}_{\mathbf{h}_{p} \mathbf{h}_{p}}=\mathrm{E}\left\{\mathbf{h}_{p} \mathbf{h}_{p}^{H}\right\}=\frac{1}{N_{s}} \mathbf{F}_{p s} \mathbf{I}_{N_{s}} \mathbf{F}_{p s}^{H} .
$$

Using this formulation, (9) can be rewritten as:

$$
\mathbf{h}_{r w}=\mathbf{F}_{u s} \mathbf{F}_{p s}^{H}\left(\mathbf{F}_{p s} \mathbf{F}_{p s}^{H}+\sigma_{w}^{2} \mathbf{I}_{N_{p}}\right)^{-1} \mathbf{h}_{l s} .
$$

We refer to this estimator as RW.

\section{Equivalence Between NRA and RW}

Comparing (7) and (12), a strong similarity can be observed. By using the SVD of the matrix $\mathbf{F}_{p s}$, the RW estimator can be expressed as:

$$
\mathbf{h}_{r w}=\mathbf{F}_{u s} \mathbf{V} \Delta_{r w} \mathbf{U}^{H} \mathbf{h}_{l s}
$$

where $\Delta_{r w}[i, j]=\frac{\lambda_{i}}{\lambda_{i}^{2}+N_{s} \sigma_{w}^{2}}$ for $i=j$, and $\Delta_{r w}[i, j]=0$ for $i \neq j$.

From inspection of (8) and (13), it is straightforward to see that $\mathbf{h}_{n r a}=\mathbf{h}_{r w}$ when $\gamma=N_{s} \sigma_{w}^{2}$. Moreover, it can be shown that this value minimizes the MSE of the estimator when no virtual subcarriers are present and regularly-spaced pilots are used, and it will be assumed for the NRA algorithm in the reminder of this paper. Therefore the full equivalence between the time-domain NRA algorithm and the frequency domain RW algorithm has been proven, assuming the same a priori knowledge available at the receiver.

\section{E. MSE of the Estimators}

The different proposed practical solutions are all covered by (7). It is therefore possible to study their respective performance by using one single closed form MSE expression. The MSE is calculated as:

$$
\operatorname{MSE}\left\{\mathbf{h}_{n r a}[n]\right\}=\mathrm{E}\left\{\left|\mathbf{h}[n]-\mathbf{h}_{n r a}[n]\right|^{2}\right\} .
$$

Using (7), the MSE for the $n^{\text {th }}$ subcarrier becomes:

$$
\operatorname{MSE}\left\{\mathbf{h}_{n r a}[n]\right\}=\mathbf{M}[n, n]
$$

where

$$
\mathbf{M}=\mathbf{F}_{u s}\left[(\mathbf{I}-\mathbf{A}) \mathbf{R}_{\mathbf{g}_{s} \mathbf{g}_{s}}(\mathbf{I}-\mathbf{A})+\sigma_{w}^{2} \mathbf{A}^{2} \mathbf{T}^{-\mathbf{1}}\right] \mathbf{F}_{\mathbf{u s}}^{\mathbf{H}} .
$$

In the previous equation, $\mathbf{A}=(\mathbf{T}+\gamma \mathbf{I})^{-\mathbf{1}} \mathbf{T}$, where $\mathbf{T}$ is defined in (6), and $\mathbf{R}_{\mathbf{g}_{s} \mathbf{g}_{s}}$ is the autocorrelation matrix of $\mathbf{g}_{s}$. The average MSE of the estimator can consequently be defined as:

$$
\overline{\mathbf{M S E}}\left\{\mathbf{h}_{n r a}[n]\right\}=\frac{1}{\mathrm{~N}_{u}} \operatorname{tr}\{\mathbf{M}\} .
$$

Note that the MSE of the ML estimator is obtained by setting $\gamma=0$ and the MSE of the RW estimator is obtained by setting $\gamma=N_{s} \sigma_{w}^{2}$.

\section{PERFORMANCE ANALYSIS}

In the following, the performance of the estimators discussed in section III will be studied. A single input single output OFDM system with the physical layer parameters proposed for the downlink of UTRA LTE will be used [1]. The system is based on a constant subcarrier spacing of $15 \mathrm{KHz}$, with different bandwidth configurations ranging from 1,25 to $20 \mathrm{MHz}$, as shown in Table 1. The CP is assumed to be always longer than the maximum delay of the channel, and QPSK modulation is used for both pilot and data symbols. Evenly spaced pilot symbols with a spacing of 6 subcarriers are transmitted in every OFDM block.

Table 1: OFDM parameters for LTE

\begin{tabular}{|c|c|c|c|}
\hline Signal Bandwidth & Nfft & Nu & Sampling frequency \\
\hline $1.25 \mathrm{MHz}$ & 128 & 75 & $1.92 \mathrm{MHz}$ \\
\hline $2.5 \mathrm{MHz}$ & 256 & 150 & $3.84 \mathrm{MHz}$ \\
\hline $5 \mathrm{MHz}$ & 512 & 300 & $7.68 \mathrm{MHz}$ \\
\hline $10 \mathrm{MHz}$ & 1024 & 600 & $15.36 \mathrm{MHz}$ \\
\hline $20 \mathrm{MHz}$ & 2048 & 1200 & $30.72 \mathrm{MHz}$ \\
\hline
\end{tabular}

In Fig. 1, the MSE of the ML estimator depending on the maximum delay of the channel is analyzed using (17), where a sample-spaced uniform PDP and an OFDM system with an FFT size of 2048 and different number of used subcarriers have been used. The Signal-to-Noise Ratio (SNR) is set to $15 \mathrm{~dB}$ and the sampling rate is the one corresponding to the LTE $20 \mathrm{MHz}$ configuration. When all the subcarriers are used, the error of the estimate grows linearly with the channel length. When virtual subcarriers are used, however, the matrix $\mathbf{T}$ to be inverted becomes ill-conditioned after a certain channel length, yielding a large degradation of the MSE that makes the estimator unusable. The maximum channel length before the estimator becomes unstable decreases as the number of used subcarriers is reduced.

Fig. 2 depicts the same analysis for the different LTE configurations shown in Table 1, which all have the same ratio between used and virtual subcarriers. The results show that the larger the bandwidth, the smaller is the maximum length of the channel that can be estimated without suffering from the illconditioning effect. In the extreme case of $20 \mathrm{MHz}$ bandwidth, only channels with a maximum delay lower than $800 \mathrm{~ns}$ can be estimated accurately, showing that ML is not a good option for systems with large FFT sizes and virtual subcarriers.

By adding a diagonal of small values to the matrix $\mathbf{T}$, the ill-conditioning of the matrix to be inverted is avoided . This is illustrated in Fig. 3, where the MSE corresponding to each subcarrier has been represented for the ML and the NRA estimators in a LTE 2,5 MHz configuration. Only half of the bandwidth has been represented, where subcarrier 0 indicates the central subcarrier. The channel profile used is a sample spaced modified ITU Pedestrian B profile, which has a maximum excess delay of $3.7 \mu \mathrm{s}$, and the SNR has been set to $15 \mathrm{~dB}$. As can be seen, ML suffers severe degradation in the edge of the bandwidth, due to the use of virtual subcarriers. NRA significantly alleviates this problem and also achieves a better performance 


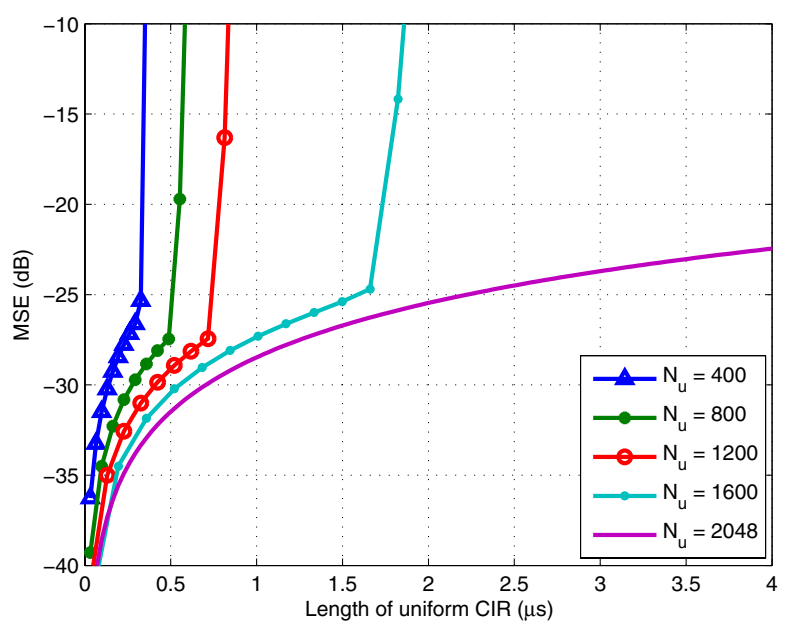

Figure 1: MSE for varying CIR length, different used bandwidths sizes, ML algorithm, $N_{f f t}=2048$ at $\mathrm{Eb} / \mathrm{No}=15 \mathrm{~dB}$

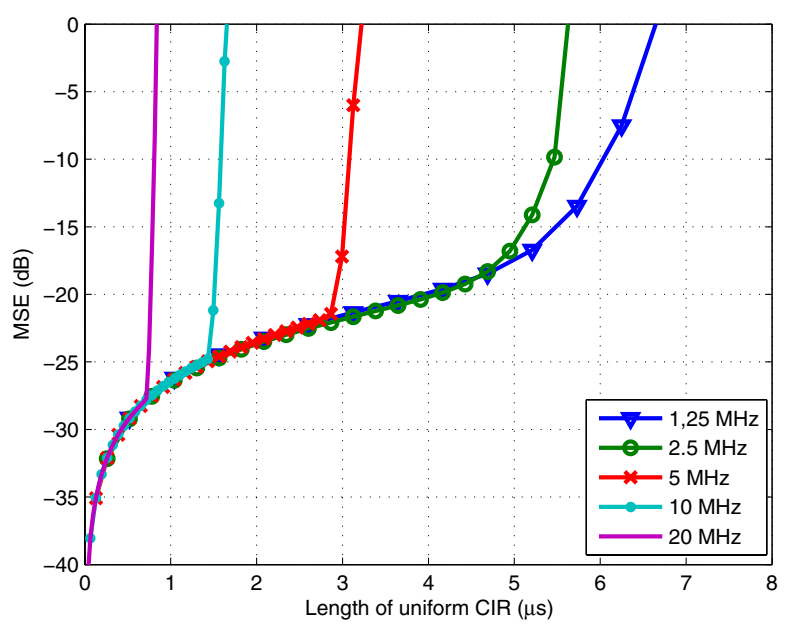

Figure 2: MSE for different bandwidths of the LTE standard and a varying uniform channel profile length, at $\mathrm{Eb} / \mathrm{No}=15 \mathrm{~dB}$. The ratio $\frac{N_{u}}{N_{f f t}}$ is fixed to 0.586

over all the bandwidth, as it makes use of the noise variance knowledge.

Finally, the Symbol Error Rate (SER) performance of ML and NRA is shown in Fig. 4, where again a sample spaced modified Pedestrian B channel profile has been used. The curves for 20 and $2.5 \mathrm{MHz}$ configurations are depicted. The results for ML show an acceptable performance for the $2.5 \mathrm{MHz}$ settings, with a degradation of around $3.5 \mathrm{~dB}$ at $1 \%$ SER with respect to perfect channel estimation. For $20 \mathrm{MHz}$, however, ML is unable to estimate the channel, as the estimator becomes numerically unstable due to the matrix inversion. NRA, on the other hand, performs better than ML in both scenarios, with a distance of $1.5 \mathrm{~dB}$ to the known channel curve. Furthermore,

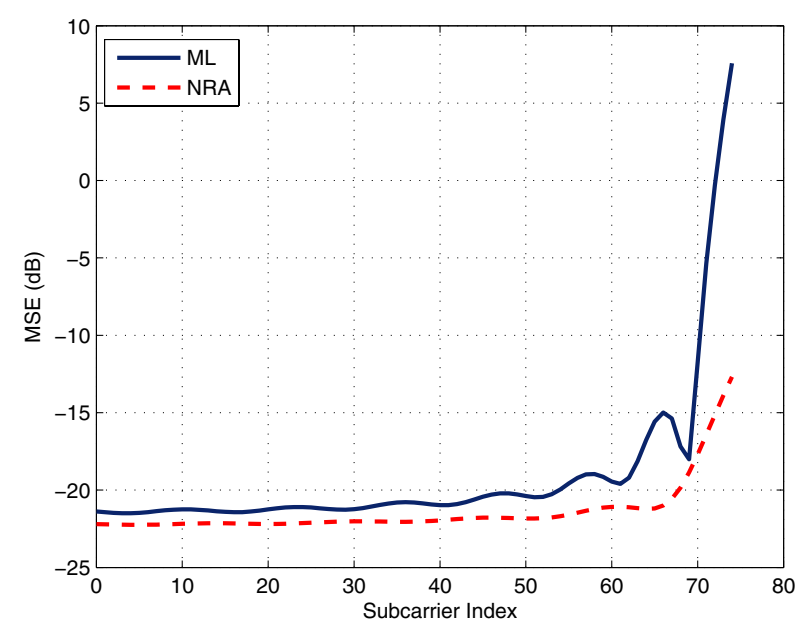

Figure 3: MSE per subcarrier for 2.5Mhz LTE settings, PedB channel, at $\mathrm{Eb} / \mathrm{No}=15 \mathrm{~dB}$

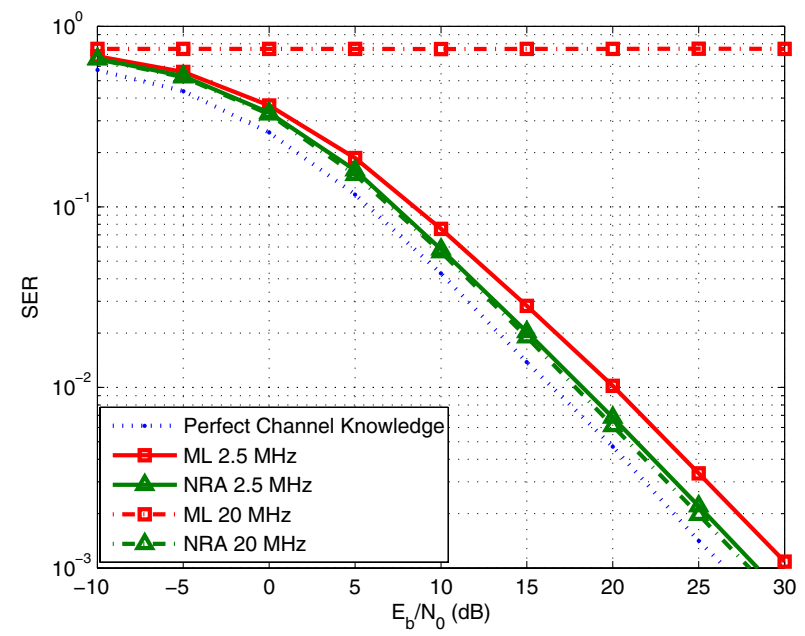

Figure 4: SER as a function of SNR for Pedestrian B channel profile, for $2,5 \mathrm{MHz}$ and $20 \mathrm{MHz}$

it exhibits total robustness to the FFT size and number of subcarriers used, turning out to be a more suitable estimator for OFDM systems in both large and small bandwidth scenarios.

\section{COMPlEXITY AND IMPLEMENTATION STRATEGY}

The optimal linear Wiener filter is discarded as it requires computation of a matrix inverse that depends on the channel statistics and is therefore computationally prohibitive for large bandwidths. Due to the high MSE's experienced by the ML algorithm, only different approaches of computing the RW or NRA algorithm are studied.

Three main implementation proposals are considered and discussed for different parameter settings. They are given as follows: 
I) A precalculated RW filter, where the filter coefficients are calculated in advance and pre-stored in memory.

II) SVD of RW where the coefficients are also pre-stored in memory.

III) FFT based NRA, where $\mathbf{F}_{u s}$ is computed by a partial input partial output FFT, as suggested in [11]. It is noted that a general analytical expression of the complexity is nontrivial in this case. For this reason the complexity of a full FFT is considered as an upper bound.

Table 2: Complexity of the Estimator

\begin{tabular}{|c|l|}
\hline Proposal & Complexity \\
\hline I & $O\left(N_{u} N_{p}\right)$ \\
\hline II & $O\left(N_{s} N_{u}+N_{s}+N_{p}^{2}\right)$ \\
\hline III & $O\left(N_{f f t} \log _{2}\left(N_{f f t}\right)+N_{s} N_{p}\right)$ \\
\hline
\end{tabular}

The orders of complexity of the different proposals are given in Table II. Practical Complex Multiply Accumulate (CMAC) operations per estimated CTF are used as complexity unit. Parameters are chosen according to the LTE settings given in Table I for the $2,5 \mathrm{MHz}$ and the $20 \mathrm{MHz}$ bandwidths. The complexity results are then shown in Fig. 5. The main complexity factor is the FFT size allowing an increased data rate. However, for the chosen solutions, the length of the CIR is critical in determining the solution with lowest complexity. When considering a small FFT size of 256, the SVD of RW has the lowest complexity if the CIR length is below 3,5 $\mathrm{s}$. On the other hand, for an FFT size of 2048, the FFT based solution has the lowest complexity for CIR lengths above 1,7 $\mu \mathrm{s}$. As the interest of complexity reduction lies in the worst case scenarios, the most promising algorithm implementation would be the one based on partial-input, partial-output FFT, where III is an upper complexity bound. As the input is of size $\mathrm{N}_{u}$ and the output of size $\mathrm{N}_{s}$ the complexity of III could be further reduced by an approximate factor of 1,5 to 2 . From Fig. 5 solution III is up to 4 times less complex than solution I and II, and with an optimized implementation [11] this could be further enhanced leading to a factor 6 to 8 times lower complexity.

\section{CONCLUSION}

Frequency direction PACE is studied for OFDM in an LTE context. In this paper we show that when virtual subcarriers are introduced, the ML time domain algorithm suffers from high MSE due to ill-conditioned matrices. The FFT size and the number of used subcarriers will determine the length of supported CIR for an ML with acceptable performance. A solution to this problem is to introduce a small value to the diagonal of the matrix to be inverted giving the NRA. We prove that there is a full equivalence between the time domain NRA and the frequency domain RW algorithm. This proof helps us to define a low complex FFT based implementation of the RW solution. Complexity evaluations show that this solution has significantly lower complexity than the classical implementations by SVD in the case of large FFT sizes.

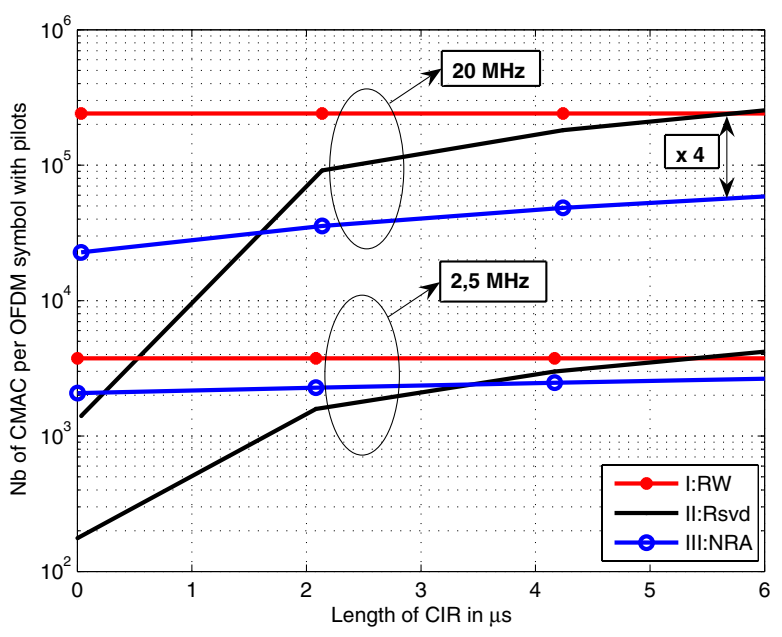

Figure 5: Complexity in CMAC of different implementations for LTE settings in both $2,5 \mathrm{MHz}$ and $20 \mathrm{MHz}$

\section{ACKNOWLEDGMENT}

The authors would like to thank Infineon Technologies Denmark and Benny Vejlgaard for their advice and financial support, thanks to whom this work was possible.

\section{REFERENCES}

[1] 3GPP TR 25.814 V7.1.0 (2006), "Physical Layer Aspects for Evolved Universal Terrestrial Radio Access (UTRA)"

[2] P. Hoeher,S. Kaiser and I. Robertson, "Two-dimensional pilot-symbolaided channel estimation by Wiener filtering," in Proc. Int. Conf. Acoustics Speech and Signal Processing (ICASSP), pp. 1845-1848, 1997.

[3] J.-J. van de Beek,O. Edfors, M. Sandell, S. K. Wilson and P.O. Borjesson, "On channel estimation in OFDM systems," in Proc. IEEE VTC'96, pp. 815-819, Nov. 1996.

[4] R. Negi and J. Cioffi, "Pilot tone selection for channel estimation in a mobile OFDM system," in IEEE Trans. Consumer Electron., vol. 44, pp. 1122-1128, Aug. 1998.

[5] L. Deneire, P. Vandenameele, L. van der Pierre, B. Gyselinckx and M. Engels, "A low complexity ML channel estimator for OFDM," in IEEE Trans. Commun, vol. 51, no.2, pp. 135-140, Feb. 2003.

[6] M. Morelli and U. Mengali, "A comparison of pilot-aided channel estimation methods for OFDM systems," in IEEE Trans. Signal Proc., vol. 49, pp. 3065-3073, December 2001.

[7] H. Schmidt, V. Kuhn, K.D. Kammeyer, R. Rueckriem and S. Fechtel, "Channel tracking in wireless OFDM systems," in SCI 2001, Orlando, Florida, USA, 22-25 July 2001.

[8] M.H. Hsieh and C.H. Wei, "Channel estimation for OFDM systems based on comb-type pilot arrangement in frequency selective fading channels," IEEE Trans. Consumer Electronics, vol.44, pp. 217-225, Feb. 1998.

[9] O. Edfors, M. Sandell, J. van de Beek, S.K. Wilson and P.O. Borjesson, "OFDM channel estimation by singular value decomposition," in IEEE Trans. Commun., vol. 46, pp. 931-939, July 1998.

[10] Y. Li, L.J. Cimini, and N.R. Sollenberger, "Robust channel estimation for OFDM systems with rapid dispersive fading," in IEEE Trans. Commun., vol. 46, pp. 902-915, July 1998.

[11] T.V. Sreenivas and P.V.S. Rao, "High-resolution narrow-band spectra by FFT prunning," in IEEE Trans. on Acoustics Speech and Signal Processing, vol. assp-28, pp. 254-257, No.2, Apr. 1980. 